\title{
Clinical Audit of Urosepsis Treated in a Malaysian Surgery Unit
}

\author{
Henry Tan Chor Lip,2,3, Tan Jih Huei1,2,3 and Tuan Nur' Azmah Tuan Mat1
}

\begin{abstract}
This is a retrospective audit of all patients admitted to the surgical unit for urosepsis from June 2014 to June 2015 at the General Surgery Unit of Hospital Sultan Ismail, Malaysia. Demographics, comorbidities, presenting symptoms, length of stay (LOS), mortality, and associated risk factors were recorded. There were 35 patients treated for urosepsis with a male preponderance of $21 / 35$ patients. Hypertension $(n=18)$ and diabetes $(n=10)$ were the two most common comorbidities. There were five deaths (mortality rate $=14.3 \%$ ) and the main bacterium cultured was Escherichia coli. Age $>65$, presence of underlying comorbid disease, presence of cancer, urine and blood culture positivity had higher incidence of death but were not statistically significant for urosepsis mortality. However, this result is limited by the small sample size and single centre retrospective data.
\end{abstract}

Key Words: Urinary tract infection, Sepsis, Surgery.

Urosepsis and sepsis incidents were reported to be increasing over recent years. Urosepsis is defined as sepsis caused by urinary tract infection, which is also a systemic response. ${ }^{1}$ Often, $20-30 \%$ of the infectious foci in septic patients originates from the urogenital tract. The manifestation of urosepsis is wide, from simple bacteriuria to severe sepsis and septic shock when presented late. ${ }^{2}$ Diagnosis is often done by assessing for urinary tract signs and symptoms (flank pain, costovertebral tenderness, dysuria, urinary retention, prostatic or scrotal pain) with bacteriuria. ${ }^{3}$ Despite modernisation of medical treatments (antibiotics) and urosurgical techniques, mortality from urosepsis still remains static at $28.3-41.1 \% .4$ Most available publications on urosepsis were analysed mainly on European and US populations. ${ }^{5,6}$ In Malaysia, there are only 13 government centres which provide specialised urology services. With a population of 30.3 million, most Malaysians, seeking emergency healthcare treatment, will have a higher probability of being treated in a general hospital without urology speciality. In addition, there is no Malaysian data published before to look at the disease incidents in a general surgery unit treating urosepsis.

Therefore, the objective of this article is to describe the morbidity, mortality trends, and mortality predictors of

\footnotetext{
1 Department of General Surgery, Hospital Sultanah Aminah, Johor Bahru, Malaysia

2 Pusat Perubatan Universiti Kebangsaan Malaysia, Cheras, Malaysia

3 Clinical Research Centre, Hospital Sultan Ismail, Johor Bahru, Malaysia

Correspondence: Dr. Henry Tan Chor Lip, Department of General Surgery, Jalan Abu Bakar, Masjid Sultan Abu Bakar, 80000 Johor Bahru, Johor, Malaysia

E-mail: relos1402@gmail.com

Received: September 19, 2017; September 28, 2018.
}

urosepsis patients treated in a Malaysian general surgery department.

This is a retrospective study of all adult surgery patients who were admitted at Hospital Sultan Ismail (HSI) for urosepsis. All patients treated by the General Surgery Department from June 2014 to June 2015 were included. Patients' registration numbers were traced from the daily admission census and parameters of interests (age, gender, race, presenting complain, underlying comorbidities, urine culture and blood culture results and mortality) was extracted from the hospital's computerised database.

Data was collected retrospectively, based on patients' records. As this was done anonymously, informed consent was not required by Malaysian Ministry of Health Research Ethics Committee. Data was retrieved by using the hospital's computerised database THIS (Total Hospital Information System). Analysis was done using SPSS (Statistical Package for the Social Sciences) for Windows version 16.0 (SPSS Inc., Chicago, USA). Descriptive data were expressed as median with interquartile value for continuous variables, frequencies with percentages for categorical variables. Logistic regression was done and $p \leq 0.05$ is considered significant.

Records for patients with the ICD-10-CM A41.9 diagnosis from June 2014 till June 2015 were screened, of which 35 patients fulfilled the inclusion and exclusion criteria. The study population had a male preponderance of 21 male patients to 14 female patients with median age of 64 (51-73) years. Median length of stay was 5 (3-7) days. There were 5/35 deaths accounting for a mortality rate of $14.3 \%$. Majority of races with urosepsis correspond to the local demography of Malaysia with the Malay and Chinese being the majority. Most of the patients with urosepsis presented with chief complaints of fever and hematuria. More than half of the sample population had comorbidities of either hypertension 18 $(51.4 \%)$ or diabetes $10(28.5 \%)$ as seen in Table I. 
Table I: Patients' demographics and characteristics of the study population.

\begin{tabular}{|c|c|}
\hline Variable & All patients $(n=35)$ \\
\hline Age* $^{*}$ & $64(51-73)$ \\
\hline \multicolumn{2}{|l|}{ Gender } \\
\hline Male & $21(60 \%)$ \\
\hline Female & $14(40 \%)$ \\
\hline LOS $^{*}$ & $5(3-7)$ \\
\hline \multicolumn{2}{|l|}{ Race } \\
\hline Malay & $18(51.4 \%)$ \\
\hline Chinese & $14(40 \%)$ \\
\hline Indian & $1(2.9 \%)$ \\
\hline Others & $2(5.7 \%)$ \\
\hline \multicolumn{2}{|l|}{ Presenting complaint } \\
\hline Hematuria & $15(42.9 \%)$ \\
\hline Dysuria & $10(28.6 \%)$ \\
\hline Urinary retention & $9(25.7 \%)$ \\
\hline Pyuria & $7(20 \%)$ \\
\hline Fever & $22(62.9)$ \\
\hline \multicolumn{2}{|l|}{ Underlying comorbidities } \\
\hline Hypertension & $18(51.4 \%)$ \\
\hline Diabetes & $10(28.5 \%)$ \\
\hline Cerebrovascular disease & $5(14.3 \%)$ \\
\hline Renal disease & $3(8.6 \%)$ \\
\hline Ischemic heart disease & $2(5.7 \%)$ \\
\hline \multicolumn{2}{|l|}{ Pre-existing urinary tract disorders } \\
\hline Benign prostatic hyperplasia & $3(8.6 \%)$ \\
\hline Urolithiasis & $4(11.4 \%)$ \\
\hline Prostate cancer & $2(5.7 \%)$ \\
\hline Bladder cancer & $1(2.9 \%)$ \\
\hline
\end{tabular}

Table II: Risk factors of urosepsis comparing survivors and nonsurvivors.

\begin{tabular}{lccc}
\hline Risk factors & $\begin{array}{c}\text { All episodes } \\
(\mathrm{n}=35)\end{array}$ & $\begin{array}{c}\text { Survivors } \\
(\mathrm{n}=30)\end{array}$ & $\begin{array}{c}\text { Non-survivors } \\
(\mathrm{n}=5)\end{array}$ \\
\hline Age $<65$ years & $18(51.4 \%)$ & $16(53.3 \%)$ & $2(40 \%)$ \\
Age $\geq 65$ years & $17(48.6 \%)$ & $14(46.7 \%)$ & $3(60 \%)$ \\
No comorbid risk factor & $10(28.6 \%)$ & $8(26.7 \%)$ & $2(40 \%)$ \\
Comorbid risk factor $\geq 1$ & $25(71.4 \%)$ & $22(73.3 \%)$ & $3(60 \%)$ \\
Presence of cancer & $9(25.7 \%)$ & $6(20 \%)$ & $3(60 \%)$ \\
Urine culture negative & $10(28.6 \%)$ & $7(23.3 \%)$ & $3(60 \%)$ \\
Urine culture positive & $20(57.1 \%)$ & $19(63.3 \%)$ & $1(20 \%)$ \\
Blood culture negative & $15(42.9 \%)$ & $13(43.3 \%)$ & $2(40 \%)$ \\
Blood culture positive & $8(22.9 \%)$ & $6(20 \%)$ & $2(40 \%)$ \\
Urine and blood culture negative & $25(71.4 \%)$ & $22(73.3 \%)$ & $3(60 \%)$ \\
Urine and blood culture positive & $4(11.4 \%)$ & $3(10 \%)$ & $1(20 \%)$ \\
\hline
\end{tabular}

Growth of Escherichia coli were seen in four patients in both urine and blood cultures. There were also positive urine cultures of Klebsiella pneumonia (2 case), with one case each of Serratia marcescens, Proteus mirabilis and Stretococcus agalacticae. Blood cultures were positve for Entercoccus, Proteus vulgaris, Morganella morgagni and Methicillin resistant staphylococcus aureus in one case each. There were eight cases of mixed growth, considered contaminated and representing incorrectly collected urine samples. Predictors of mortality of interest in survivors and non-survivors are described in Table II. Univariate analysis did not detect any significant difference. There were five mortalities reported in our study. Four patients were identified to be having urosepsis due to obstruction uropathy secondary to benign prostatic hyperplasia and staghorn calculi that presented in irreversible septic shock (one case each), two patients had metastatic cancer (prostate, colon cancer) which were already issued not-for-activeresuscitation prior admission. One patient died due to an infected percutaneous nephrostomy insertion with multiple comorbidities of cerebrovascular and ischemic heart disease.

Within a year, the General Surgery Unit of HSI treated 35 cases of urosepsis, with a mortality rate of $14.3 \%$. Wahgenlehner et al. reported mortality rate from urosepsis in the range of $28-40 \%$ from sources which included sepsis from European and US populations. ${ }^{2,3}$ $E$. coli was the predominant cause of urosepsis with a high number of urine cultures (eight urine samples) considered contaminated with mixed growths. This may be attributed due to poor sterility during urine sampling and lack of awareness in proper handling of urine samples. Univariate analysis of risk factors of interest (advanced age $\geq 65$, presence of $\geq 1$ underlying comorbidities, presence of cancer, urine culture, and blood culture positivity) showed non-insignificant results. These results may be affected due to the small sample population and low number of deaths. Although the results generated did not yield any significant results as predictor of urosepsis mortality, it did show the epidemiologic trends to compare with populations outside Southeast Asia. More studies are required to know the risk factors and death trends in urosepsis for mortality risk counselling and disease severity triage on admission.

\section{REFERENCES}

1. Book M, Lehmann LE, Schewe JC, Weber S, Stuber F. Urosepsis: Current therapy and diagnosis. Urolodge 2005; 44:413-24.

2. Brun-Buisson $\mathrm{C}$. The epidemiology of the systemic inflammatory response. Intensive Care Med 2000; 26:64-74.

3. Munir T, Lodhi M, Hussain RM, Mubeen M. Association between periurethral colonization and subsequent bacteriuria in catheterized patients. J Coll Physicians Surg Pak 2009; 19:169-72.

4. Wagenlehner FM, Lichtenstern C, Rolfes C, Mayer K, Uhle F. Diagnosis and management of urosepsis. Int J Urol 2013; 20: 963-70.

5. Angus DC, Linde-Zwirble WT, Lidicker J, Clermont G, Carcillo J, Pinsky MR. Epidemiology of severe sepsis in the United States: analysis of incidence, outcome, and associated costs of care. Crit Care Med 2001; 29:1303-10.

6. Bjerklund Johansen TE, Cek M, Naber K, Stratchounski L, Svendsen MV, Tenke P. Prevalence of hospital-acquired urinary tract infections in urology departments. Eur Urol 2007; 51: 1100-11.

$$
\text { .......... }
$$

